\title{
The Welfare of Women Safety Based on IoT
}

\author{
${ }^{1}$ B.Narmadha, ${ }^{2}$ M.Ramkumar, ${ }^{3}$ Srinivasan $\mathrm{M},{ }^{4}$ Priyanga T, ${ }^{5}$ K.Vengatesan \\ ${ }^{1,2}$ Assistant Professor, ${ }^{3,4}$ Student \\ ${ }^{1,2}$ Department of Computer Science Engineering, \\ ${ }_{1,2,3,4}$ Knowledge Institute of Technology, Salem, India \\ ${ }^{5}$ Associate Professor, Department of Computer Engineering, \\ ${ }^{5}$ Sanjivani College of Engineering, Kopargaon
}

\begin{abstract}
Now Recent trends are opening up to the era of Internet of Things (IoT), which is the communication between Internet and the devices. This is one of the emerging technologies, from the perspective of smart technologies, which is always been expected by the forthcoming generation. This System focuses on a security system that is designed solely to serve the purpose of providing security to women so that they never feel helpless while facing such social challenges. The system consists of various modules such as Indication, Emergency, Medic care, Dietary. All these modules has different function which will help women to be feel safe and secure in our country. We propose to have arduino device using IOT, which continuously communicates with Smart phone that has access to the internet. The application is programmed and loaded with all the required data which include object detection, pressure (BP) and heartbeat. This generates a signal which is transmitted to the smart phone. The software or application has access to GPS and Messaging services which is pre-programmed in such a way that whenever it receives emergency signal, it can send help request along with the location co-ordinates to the nearby police station. For pregnancy women, this system will helpful if they have any false pain during the time of pregnancy the alert will send to their contacts, doctor and an ambulance. Also the dietary chart is available for pregnancy women so that they can take care of their health. In dietary chart the details of food that is what food they have to take and in what time is displayed as notification. If user has taken that food displayed as the notification, the user can tick the checkbox shown in the mobile. At last the report of their daily details of food is display at the day end. So this band will be very useful for women to be safe and secure.
\end{abstract}

Index Terms - Security system, GPS location, Pregnancy women, Women safety, Internet of things.

\section{INTRODUCTION}

$\mathrm{T}$ he world is becoming very unsafe to women in this society. Security for women is still a major issue as the number of crimes over women and girls is increasing day-byday. In this age of technology, mobile phone is one gadget almost everyone uses to keep in touch with family and friends. All they need is a device that can be carried around easily and worn whenever the woman feels unsafe. An advanced system can be set up to detect location and health status the person that will enable us to take action accordingly based on electronic devices such as GPS, object sensor, pulse rate sensor, heartbeat and IOT. We can make use number of sensors to precisely detect the real time situation of the women in critical abusive situations. The heartbeat of a person in such situations is normally higher which helps make decisions

along with other sensors. The idea of developing a smart device for women is that it is completely comfortable and easy to use compared to existing women safety solutions, such as: Garments, bulky belts and notorious mobile applications that are very abstract and obsolete. Smart band integrated with the smartphone has an additional advantage so as to reduce the cost of the device and also in reduced size.

\section{MATERIALS AND METHODS}

\section{A. Indication Button}

The indication button is used to send a message to the user's family and friends indicating whether the user is safe with the location or not. If the band is not with the user the object sensor will sense and send 3alert messages to the mobile. When the user is not respond for this 3 alert messages then indication will send to the user's family and friends that they are in some emergency.

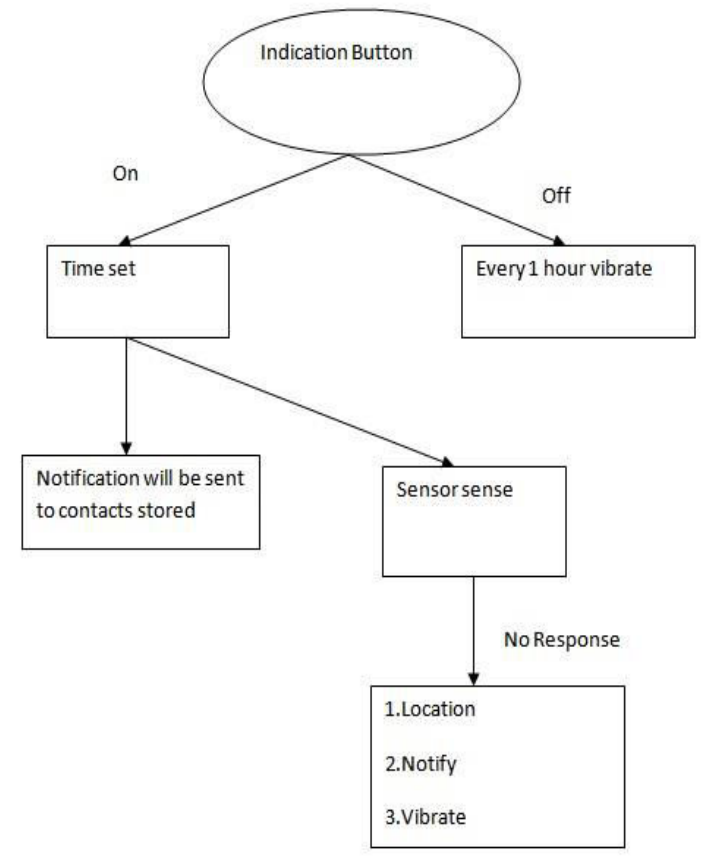

Fig.1 Indication Flowchart Diagram.

\section{B. Emergency Button}

If the women knows that they are in real danger or in emergency situation they should press the emergency button which will automatically send an emergency message based 
on the GPS location to the nearby police station so that the police can rescue the victim. The nearest location can be tracked using the GPS module. The emergency button is an electronic device designed to assist in alerting somebody in emergency situations where a threat to persons or property exists.

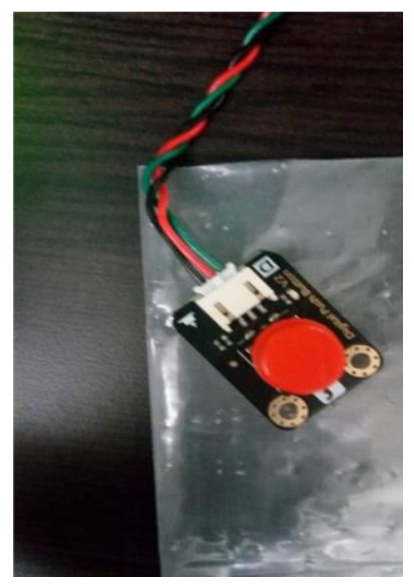

Fig. 2 Emergency Button

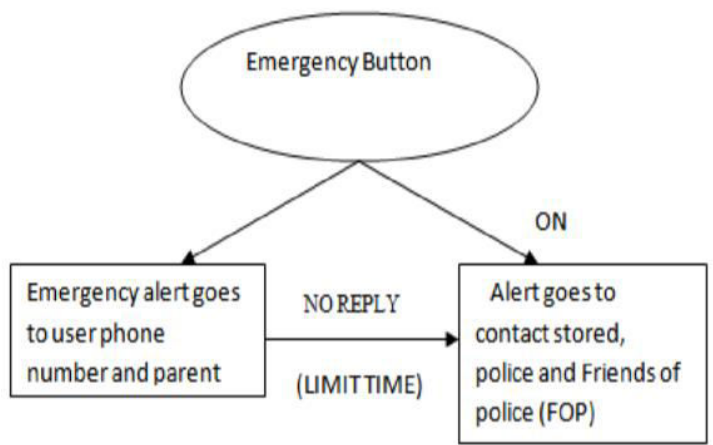

Fig.3. Medic care Flowchart Diagram

\section{Medi Care}

This is been used by the pregnant women's during their pain. Say for instance they are in the home alone, in case they have pain now a day's most of the deliveries are caesareans which cause many deaths by careless and unknown of time duration. Before pregnancy, all the women have a false pain we can't predict. In addition to this, the senior citizenship faces many problems like high pressure, unknown of time, false pain, blood clot etc., which leads to death. Our product will ensure women's safety. By clicking this button an indication will be sent to the nearest hospitals and private ambulance.

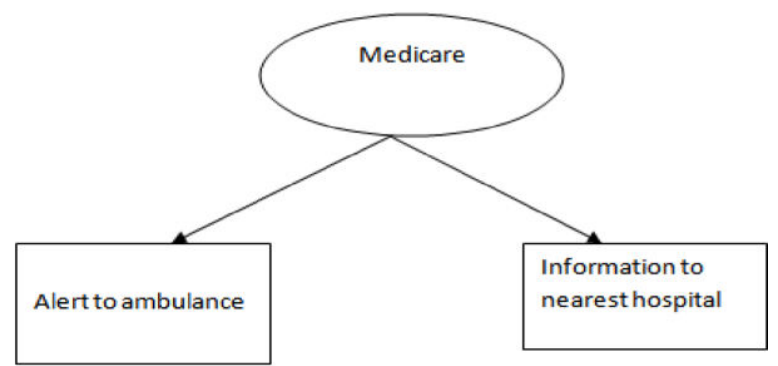

Fig.4. Medic care Flowchart Diagram

\section{Dietary}

In the mobile application there is a module for the pregnant women diet month wise. They also get indication for every hour .In case if they forget anything they will get alert. Moreover, at the day end they will get a report by calculating their heart beat, pressure and their diet on that day. In addition to this they also get month wise report so that they can have a comparison from last month.

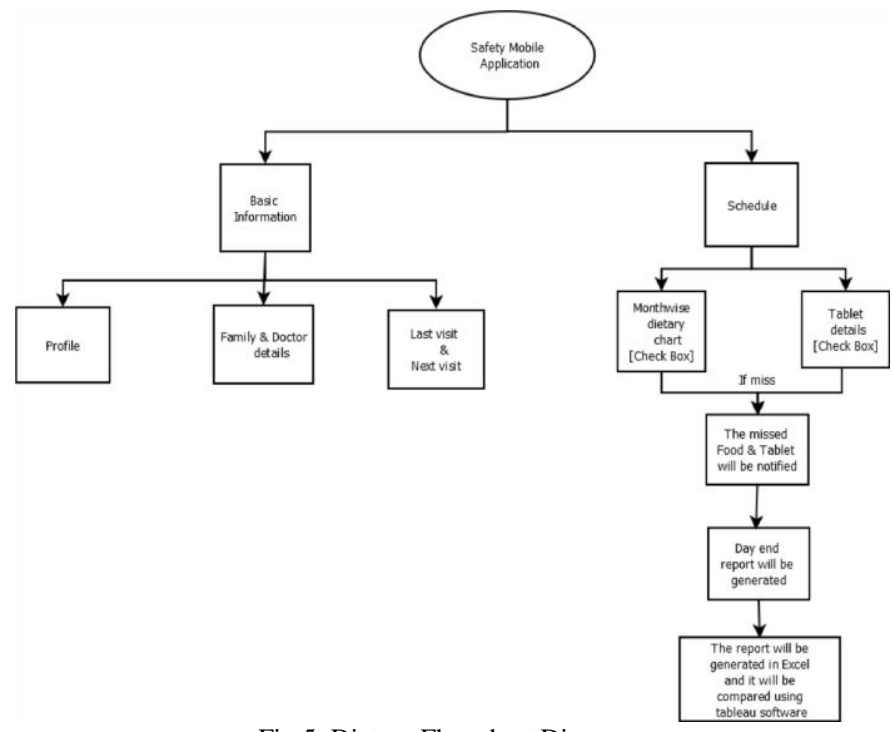

Fig.5. Dietary Flowchart Diagram

\section{E. Sensors}

A sensor is an electronic component, module, or subsystem whose purpose is to detect events or changes in its environment and send the information to other electronics, frequently a computer processor. A sensor is always used with other electronics, whether as simple as a light or as complex as a computer. In this three sensors were used, they are the followings are

1. Object Sensor

2. Pressure Detecting Sensor

3. Heart Beat Sensor

\section{Object Sensor}

The object sensor tracks whether the safety band is on the user's hand. In case, if the band is not with the user then it will send 3 alert messages to the user mobile. If the user does not give the response to the alert it directly connects to indication button and does indication process automatically. Moreover, 
the parents can proceed the emergency process. Object detection is a computer technology related to computer vision and image processing that deals with detecting instances of semantic objects of a certain class (such as humans, buildings, or cars) in digital images and videos. This sensor is analogous to human's visionary senses, which can be used to detect obstacles and it is one of the common applications in real time The goal of object detection is to detect all instances of objects from a known class, such as people, cars or faces in an image. Typically only a small number of instances of the object are present in the image, but there are a very large number of possible locations and scales at which they can occur and that need to somehow be explored. Object detection systems construct a model for an object class from a set of training examples. In the case of a fixed rigid object only one example may be needed, but more generally multiple training examples are necessary to capture certain aspects of class variability. The reasons... many, but in general they are able to learn features (usually much better than hand-crafted one's HOG, SIFT etc.) with location and slight transformation invariance (because of the convolutions and the pooling), which makes them a very attractive choice.

\section{Pressure Detecting Sensor}

A pressure sensor, as the name suggests, is a device that senses and measures pressure (usually of gases or liquids). The pressure sensor in electronic circuits is in the form of an integrated circuit that acts as a transducer, that is, it replicates (in the form of an electrical signal) the signal it receives as a function of imposed pressure. Blood pressure measurement is divided into systolic and diastolic. Systolic blood pressure is the pressure during the contraction of the heart. Diastolic blood pressure is the pressure during the relaxation phase. Blood pressure is measured in $\mathrm{mmHg}$ (millimeters of mercury), a unit of pressure. It has been shown to rise during aroused states due to the activity of the Sympathetic system (Bernardi et al. 2000). Sorvoja (2006) provides a review of practices on non-invasive blood pressure measurement and gives emphasis to three techniques: Tonometry, Pulse Transit Time (PTT) and Volume Clamp. Tonometry measures blood pressure by applying external pressure on the skin above the artery. As the artery contracts and expands due to the passage of the blood, the sensor will measure the pressure applied on the skin and give a reading of blood pressure.Atonometric sensor can be easily integrated into a wrist watch. Basically, if the blood pressure is higher, the blood takes less time to propagate into the arteries. This technique can use PPG to measure the changes in blood volume in 2 places, typically the wrist and fingertip. In a stress response, the heart is one of the first organs to react, with an increase in the bpm determined by a withdrawal of Parasympathetic influence and increased influence of the Sympathetic system. To best time to do it is in the morning, just after waking up from a good night of sleep.

\section{Heart Beat Sensor}

A person's heartbeat is the sound of the valves in his/her's heart contracting or expanding as they force blood from one region to another. The number of times the heart beats per minute (BPM). Is the heart beat rate and the beat of the heart that can be feel in any artery that lies close to the skin is the pulse. Heart beat can be checked manually by checking one's pulses at two locations - wrist (the radial pulse) and the neck (carotid pulse). The procedure is to place the two fingers (index and middle finger) on the wrist (or neck below the windpipe) and count the number of pulses for 30 seconds and then multiplying that number by 2 to get the heart beat rate. However pressure should be applied minimum and also fingers should be moved up and down till the pulse is felt. By using a sensor heart beat can be measured based on optical power variation as light is scattered or absorbed during its path through the blood as the heart beat changes. The heart beat sensor is based on the principle of photo phlethysmography. It measures the change in volume of blood through any organ of the body which causes a change in the light intensity through that organ (a vascular region). In case of applications where heart pulse rate is to be monitored, the timing of the pulses is more important. The flow of blood volume is decided by the rate of heart pulses and since light is absorbed by blood, the signal pulses are equivalent to the heart beat pulses. There is already a BAND which consists of Arduino Board, GSM/GPS modules, screaming alarm and pressure sensors. When the threshold of the heart beat sensor crosses, the device will get activated automatically. Immediately the location of the victim will be tracked with the help of GPS and emergency messages will be sent to three contacts and one to police control room every two minutes with updated location. The screaming alarm unit will be activated and will send out sirens to call out for help. The system is also capable to generate an electric shock to harm the attacker which may help the victim to escape.

\section{PROPOSED SYSTEM}

To overcome all these issues we are glad to introduce our product called SAFETY BAND. This band looks like an ordinary band with extra-ordinary features of emergency alert, ease notify, location tracker, etc., At first connecting the band with our mobile via Bluetooth, so there is a connectivity between the band and our mobile, so they can get notification to their mobile in any emergency situation and about the dietary process. They can also add some of their contacts in their mobile to get an alert at any emergency situation.

And also they can add their doctor"s mobile number to get an alert in the case of pregnancy pain. The dietary process of the pregnant women is also included with this. This will be helpful to maintain their dietary chart during pregnancy. Every day they get notification about what food have to take at that particular time in a day. And they have to make the tick if they had taken their food which is given in notification and they should not make the tick if they had not taken their food. Moreover, at the day end they will get a report by calculating their heart beat, pressure and their diet on that day. In addition to this they also get month wise report so that they can have a comparison from last month.

In this three sensors were used they are object sensor, heartbeat sensor, pressure detecting sensor. The object sensor is used to find the exact location of the user at the emergency situation, that location was detected by the object sensor and that location is send to their contacts in the case of emergency. The heartbeat sensor is mainly used to find their heartbeat rate 
per minute and the beat of the heart can be felt in an artery that lies close to the skin are the pulse. This measures the change in volume of blood through any organ of the body which causes a change in the light intensity through that organ. In case of applications heart pulse rate is to be monitored. A pressure sensor is a device that senses pressure of the human beings. Having a high blood pressure, also called hypertension, that is not under control can result in heart problems, stroke, and other medical conditions. Normal blood pressure is a systolic pressure of less than 120 millimeters of mercury $(\mathrm{mm} \mathrm{Hg})$ and a diastolic pressure of less than $80 \mathrm{~mm}$ $\mathrm{Hg}$, or $120 / 80 \mathrm{~mm} \mathrm{Hg}$, according to the American Heart Association (AHA). When there is a change in the blood pressure or the increasing the level of their pressure, the notification will sent to the doctor. In case the band was lost, the user can deactivate the band through their mobile.so no one can use the band illegally. . So, this SAFETY BAND is an innovative idea for women security which can be used like ordinary band and it is very easy to operate at the time of danger.

\section{CONCLUSION}

Women face several problems in the society, at the time of danger they can't take their mobile and call the police or to someone. So, this SAFETY BAND is an innovative idea for women security which can be used like ordinary band and it is very easy to operate at the time of danger. At present many of the girls and women having more health issues that are arising. They are not taking proper intake of health foods this leads to cause problem during their pregnancy time by using this womb band we can reduce many issues and also such features are added in the womb band for make the pregnant lady in the comfort zone.

\section{REFERENCES}

[1] Chennur, P. and Patil, S. (2015) "Self Defense System For Women Safety With Location Tracking And SMS Alerting Through GSM Network" Volume 4, Issue 5.

[2] Mr.Vasim K. Ustad, Prof.A.S.Mali, Mr.Suhas S.Kibile "Zigbee Based Wireless Air Pollution Monitoring System Using Low Cost And Energy Efficient Sensors" International Journal of Engineering Trends and Technology (IJETT) - Volume 10 Number 9 - Apr 2014.

[3] Gowtham. Sarella Mrs. Dr. Anjali. K. Khambete "Ambient Air Quality Analysis Using Air Quality Index - A Case Study Of Vapi "IJIRST International Journal for Innovative Research in Science \& Technology, Volume 1, Issue 10, March 2015

[4] Sagar, Dr Rajesh Goel "Wireless Sensor Network Deployed for Air Pollution Monitoring System in any Area" SSRG International Journal of Electrical and Electronics Engineering (SSRG-IJEEE) - EFES April 2015.

[5] Anil.H.Sonune, S.M.Hambarde "Monitoring And Controlling Of Air Pollution Using Intelligent Control System" International Journal of Scientific Engineering and Technology Volume No.4 Issue No5, pp: 310-313.

[6] Kalaivanan M., and K. Vengatesan.: Recommendation system based on statistical analysis of ranking from user. International Conference on Information Communication and Embedded Systems (ICICES), pp.479484, IEEE, (2013).

[7] Vengatesan K., Mahajan S.B., Sanjeevikumar P., Mangrule R., Kala V., Pragadeeswaran (2018) Performance Analysis of Gene Expression Data Using Mann-Whitney U Test. In: Konkani A., Bera R., Paul S. (eds)
Advances in Systems, Control and Automation. Lecture Notes in Electrical Engineering, vol 442. Springer, Singapore.

[8] Vengatesan K., Mahajan S.B., Sanjeevikumar P., Moin S. (2018) The Performance Enhancement of Statistically Significant Bicluster Using Analysis of Variance. In: Konkani A., Bera R., Paul S. (eds) Advances in Systems, Control and Automation. Lecture Notes in Electrical Engineering, vol 442. Springer, Singapore

[9] B.Narmadha, M.Ramkumar, K.Vengatesan, M.Srinivasan, "Household Safety based on IOT", International Journal of Engineering Development and Research (IJEDR), ISSN:2321-9939, Volume.5, Issue 4, pp.1485-1492, December 2017

[10] Snehal Sirsikar, Priya Karemore "Review Paper on Air Pollution Monitoring system" International Journal of Advanced Research in Computer and Communication Engineering Vol. 4, Issue 1, January 2015.

[11] Gaikwad Varsha Bhagwan, Puranik V.G” Real Time Air Pollution Monitoring Using Mobile Phone" International Journal of Innovative Research in Science, Engineering and Technology An ISO 3297: 2007 Certified Organization Volume 3, Special Issue 4, April 2014. 